\title{
Special issue on Controlo2012: new applications of artificial neural networks in modeling and control
}

\author{
Fernando Morgado-Dias • Ana Antunes • \\ José Vieira
}

Published online: 15 August 2013

(C) Springer-Verlag London 2013

The Portuguese Conference on Automatic Control, Controlo for short, started in 1994 and had its first edition in Lisbon. It is organized by the Portuguese Association of Automatic Control, which is the Portuguese National Member Organization of the International Federation of Automatic Control (IFAC). The conference has been organized every two years and attracts researchers and practitioners in the area of Automatic Control, in a broad sense, from universities and companies around the world, with a strong emphasis in the Portuguese, Spanish, and Brazilian community. In 2012, the 10th edition of the conference took place in Funchal, Madeira, Portugal, and received a large number of submissions and participants representing all the continents.

Since the very beginning of the uprising of Artificial Neural Networks (ANN) with the remarkable milestones such as the first artificial neural network model developed by McCulloch and Pitts [1], the initial Perceptron model by Rosenblatt and Wightman [2], the limitations of the perceptron presented by Minsky and Papert [3], or the proof that ANN could be used as universal approximator by Cybenko [4], among many others, ANN have found

F. Morgado-Dias ( $\square)$

Madeira Interactive Technologies Institute and Competence Center of Exact Science and Engineering, University of Madeira, 9000-390 Funchal, Portugal

e-mail: morgado@uma.pt

A. Antunes

Escola Superior de Tecnologia de Setúbal, Polytechnic Institute of Setúbal, 2910-761 Setúbal, Portugal

J. Vieira

Escola Superior de Tecnologia de Castelo Branco, Unidade Técnico Cientifica de Engenharia Electrotécnica e Industrial, Av. Empresário, 6000 Castelo Branco, Portugal application throughout the widest range of objectives and areas. The objectives go from fraud detection to forecasting while the areas can cover uncorrelated subjects such as the social areas or engineering.

The area of Automatic Control is a natural test bed for Artificial Intelligence tools, in particular Artificial Neural Networks and Fuzzy Logic that can be used for Modeling and Control in the most diverse applications.

This special issue presents a sample of the application of these tools to Modeling and Control with nine papers:

- Generation of artificial neural networks models in anticancer study, which presents a health application to determine the relationships between different compounds' structure and their anticancer activity and uses ANN for the compounds classification.

- Comparison of optical performance monitoring techniques using artificial neural networks, which reports a telecommunications comparison of different techniques for monitoring using optical fibers.

- A survey of software and hardware use in artificial neural networks, which shows and analyses the results of a survey to the ANN community inquiring about software and hardware use for training and implementing ANN in hardware.

- Low-resource hardware implementation of the hyperbolic tangent for artificial neural networks, which reports a study on how to implement in hardware the common ANN activation function, hyperbolic tangent, with polynomials while minimizing the resources necessary and introducing the smallest error possible when compared to the software alternative.

- A survey of artificial neural network training tools, which presents a digest of the training tools for ANN that the user can find today, along with license, 
algorithms, operating system, and other requirements necessary to use these tools.

- Fuzzy controllers gains tuning: a constrained nonlinear optimization approach, which presents a methodology for tuning the gains of fuzzy proportional-integral controllers where the closed-loop control system performance is explicitly taken into account.

- Assessment of data-driven modeling strategies for water delivery canals, which is a modeling application for water canals and makes use and compares ANN and other techniques such as Composite Local Linear Models and Fuzzy Models to capture all significant dynamics for the canal system.

- Tracking in scale quad-rotors through adaptive augmentation, which presents an application to flight control by using a conventional PD controller conceived mainly for hovering, with an ANN using realtime tuning in an inner-outer loop combined architecture to account for model inversion error cancelation, issued in the feedback linearization process.

- Analysis of electricity bills using visual continuous maps, which presents an application of ANN to analyze electricity bills of the different buildings at the University of Leon, by using visual continuous maps and dimensionality reduction.

These papers were selected based on the recommendation of the conference organizers, reviewers, and guest editors. The selected paper's authors were invited to resubmit an extended version of their conference papers for this special issue of Neural Computing and Applications. All these journal articles went through a rigorous review procedure before being accepted for publication.

The guest editors would like to thank all authors for their precious contributions to this special issue. We also would like to express our sincere appreciation to the members of the International Program Committee of Controlo2012 and all the reviewers for their time and efforts that resulted in a better set of selected papers, and to the Neural Computing and Applications editorial board for their support in the organizing procedure. In addition, we would also like to thank Dr. John MacIntyre, Editor-InChief of the Neural Computing and Applications.

We hope you will enjoy reading this special issue.

\section{References}

1. McCulloch WS, Pitts W (1943) A logical calculus of the ideas immanent in nervous activity. Bulletin Math Biophys 5:115-133

2. Rosenblatt F (1958) The perceptron: a probabilistic model for information storage and organization in the brain. Psychol Rev 65:386-408

3. Minsky M, Papert S (1969) Perceptrons. MIT Press, Cambridge

4. Cybenko G (1989) Approximation by superposition of a sigmoidal function. Math Control Signals Syst 2:492-499 